\title{
Development of a Robot-Based Environment for Training Children with Autism
}

\author{
Emilia I. Barakova ${ }^{1}$, Min-Gyu Kim ${ }^{1}$, and Tino Lourens ${ }^{2}$ \\ ${ }^{1}$ Eindhoven University of Technology P.O. Box 513, Eindhoven, The Netherlands \\ \{e.i.barakova,m.kim\} @tue.nl \\ ${ }^{2}$ TiViPE, Kanaaldijk ZW 11, Helmond, The Netherlands \\ tino@tivipe.com
}

\begin{abstract}
This study is done as a part of design-research processes that aims to co-create technology supported robot centered therapy environment for autistic children. We attempt to evaluate to which extent the therapists who perform behavioral training of children with autism can be supported by robot technology in the process of therapy content creation and training. First, we feature a robotcentered environment that is technically designed to decrease the complexity of programming dynamic, synchronous and parallel interactive robot behavior to a level compatible with content creation. Afterwards, we apply the Cognitive Dimensions Framework (CDF) approach for evaluation of the usability of this environment that is employed to control a robot interacting with children with Autism Spectrum Disorders (ASD). A pilot test with therapists of two clinics followed by a test with adolescents with autism was performed. Participants in the pilot test performed tasks according to the different types of user activity in the $\mathrm{CDF}$, and answered a questionnaire corresponding with the different dimensions. The results show negative attitude towards one particular dimension, but also high scores in other dimensions. As an additional validation of the usability of the environment, 9 adolescents with ASD could also create robot scenarios. We interpret these results as follows. In general, the therapists and autistic adolescents could program relatively simple behavioral scenarios with robots. However, we need to further explore whether assembling and executing of more complex robot scenarios such as programming of dynamic real-life behaviors and task scheduling is possible by end-users.
\end{abstract}

Keywords: autism spectrum disorder, cognitive dimensions framework, co-creation of contents, robot assisted autism therapy.

\section{Introduction}

There is increasingly more evidence that robots can be beneficial in behavioral training of autistic children. Robots with different embodiment and level of anthropomorphism have been used to train shared gaze and joint attention abilities [1-13,30]. Attempts have been made to use robots to improve imitation and turn-taking skills [4-9], to teach facial and body emotions [10] to enhance nonverbal and verbal skills 
27 and initiate social interaction [6], [11-13]. Even though some of these studies are successful, it is challenging to implement the robots in daily training practice. We investigate the problem of how to create a robot-centered learning environment that can be used by therapists/caregivers to support the training of children with autism.

At this stage of deployment of robots in autism research, we consider the experimenters with psychology or clinical background, therapists and caregivers as a primary user of robot technology, who will take active part in creation of robot-mediated training. So we develop training environment to serve primary the need of these users. Therefore a robot -centered training environment needs to have first, a sufficient number of robot behaviors that are useful for training, and second, an interface for easy (re-)design, personalization or adaptation of the robot training scenarios and their execution.

The issue of creating a meaningful training sessions with a robot has been addressed previously in [14]. However, in this study the therapist was a knowledge provider and he/she was not meant to create or execute robot scenarios. Robins and colleagues 28 presented a set of ten play scenarios for robot-assisted play for children with special needs; however therapist were not meant to create their own scenarios. The "Therapist-in-the-loop" approach was proposed by Colton and colleagues in [15], in which the authors attempt to engage the child and facilitate social interactions between the child and a team of therapists. We engaged the therapists in a co-creation process for the development of scenarios that they would like to use as an augmentation to their practice. The current research has the far reaching goal to make the therapists able to program, adapt and control the robot in an end-user way, relaying on co-created crucial mass of behaviors and scenarios.

To direct this process, we have chosen a specific training framework. The existing training practices for ASD address specific skills or behaviors like language and communication skills, problem solving skills, daily living skills or socially adaptive behaviors [16], [17] and use huge variety of approaches and underlying theories. We have chosen Applied Behavioral Analysis (ABA) framework, which stimulates desirable actions by children through structural positive reinforcement [18]. Pivotal Response Training (PRT) in particular, as part of ABA, introduces the so called pivotal areas to point out clusters of behaviors which, when targeted during an intervention, also lead to improvements in other behaviors of the children. So far, research has focused on five pivotal areas: responding to multiple cues, motivation, selfmanagement, self-initiations during social interactions and empathy [19], [20]. This therapy requires natural settings. Using a robotic agent to perform different scenarios with a trainer and a child is not a natural circumstance, as is required for PRT. However, we do find these techniques from ABA, and the prompts used in PRT to be a promising course to follow, since they need to be delivered in a consistent and structural manner, which is especially suitable to be done with a robot.

In an attempt to make the robot centered environments a part of the clinical testing and practice, the therapists should be able to use the robot and eventually adapt/personalize scenarios by themselves. Therefore, in addition to our efforts to create useful scenarios, it will be necessary that the therapists can change their scenarios "on the fly", i.e. they need to have robot programming skills. For this purpose we 
have developed an existing end-user programming environment for the control of a NAO robot and this paper offers a qualitative pilot study of whether the therapists can work with it. The term 'therapists' is used as an umbrella term, covering actual therapists, that perform the training and child psychologists that create the training programs.

In addition, a test has been made to check whether high IQ adolescents with autism could create robot behaviors with this environment.

In this paper, section 2 elaborates on the design of the learning environment that has the affordances of easy creation of interactive and dynamic behaviors by endusers. Section 3 describes the user testing method for the pilot test, section 4 reports the results of testing with both therapists and with adolescents with autism, and section 5 draws conclusions and offers a discussion our findings.

\section{Creation of Robot Centered Training Environment}

A robot centered learning environment needs to provide possibilities for natural interaction with the robot as well as an interface tools for the therapists/experimenters of this environment to make use these possibilities. In the domains of physical therapy and mental potential development, the so called "dual user" problem exists: a domain specialist that uses the robot as a tool to augment his/her practice, and the client or patient that is traditionally served by the domain specialist and who is now (partially) served by a robot 23 . First we need to crate affordances for use by therapists as users that will enable the uptake of robots in therapy.

Therapists cannot be expected to have programming experience, or knowledge on robotics. In addition, only a robot platform is not sufficient to make them use robots. We aim to enable the therapists with a process and a tool that will help them to build naturalistic scenarios quickly; the scenarios should be accurately performed by the robot (errors during the scenario will sabotage the learning moment for the child); in addition, the scenarios has to be fitted to the individual requirements of the child (scenarios should be easy to adapt).

For this purpose we combined a commercially available and affordable robot NAO with the TiViPE graphical programming software integrator platform [21, 23].

\subsection{Hardware Platform}

NAO robot from Aldebaran robotics is a $58 \mathrm{~cm}$ tall walking robot, having 25 mechanical degrees of freedom, and has digital cameras, speakers and microphones, different touch sensors and wireless communication capabilities. Using these sensors it can engage in interactive behavior through movement, speech, different LEDs in the face and body, and touch.

Moreover, we do not expect this specific choice of robot to be fixed forever. The TiViPE software platform is set up in such a way that different robots can be fitted to it in the future, as this field is still rapidly progressing, and more robots will become available in the future, a textual robotic language of small sets of meta commands 
such as (move, say) is being used which have to be adapted to the actuating capabilities of the used robot.

\subsection{User-Centered Software Tool}

\section{A. Drag and drop interface that incorporates synchronization, parallelism, be- havioral dynamics}

Graphical programming interfaces are generally known to improve the usability of programming tools. They usually incorporate an easy to use drag and drop interface. The NAO robots are shipped with a graphical programming environment called Choreograph. Commonly the robot manufacturers provide a software environment to control robots, mostly tailored to the specification of the robot (for instance Choreograph and leJOS 29). Besides that these environments are tightly coupled to a specific robot, they lack the ability of more complex control concepts, such as synchronization, parallelism, and creation of dynamic behaviors. We created the TiViPE robot controller, which by design incorporates these concepts. The other advantage of TiViPE is the easy adaptation to any robot, but also the ability to incorporate in the experimental or the training platform another sensors/ devices. This implies that computer or external camera, Kinnect sensor or other sensors can naturally be integrates to the learning environment.

The created robot behaviors are kept up to the environmental changes by a constant flow of sensory information of all robot sensors, they are available to the robot in real-time. The proposed environment uses a box-wire approach to create flowcharts with behavioral sequences. The boxes, which represent behavioral components with different levels of complexity, can be connected to other components, creating a network. Properties of a component can also be modified. Furthermore, networks of components can be merged into new components, which enable users to build complex, interactive and intelligent behaviors. By providing the therapists with a powerful set of components we expect that they can quickly setup a scenario by connecting different components into a network.

\section{B. Textual (string) language for creation and scheduling the priorities of execu- tion of parallel behaviors}

The main merit of a textual robot language is that robot actions are described in intuitive to humans terms such as move, LEDs on, etc., and these actions can be executed in a mixture of parallel and serial actions [23]. The complexity of a task such as designing parallel actions ( such as speak and move accordingly) is reduced to basic logic ( high school arithmetic) using \& character for sequential and "l" character for parallel actions. Square brackets are used to bind a set of these textual actions in order to give the priority of their execution where such is needed. Therefore, this textual language has the complexity of learning 10 robot commands and their parameters, and requires understanding of high school arithmetic for scheduling of the robot behaviors. One can easily understand how to schedule commands that come in parallel, or needs to wait until the last of several parallel commands have been completed. Very important feature by this parallel processing is the synchronization between parallel processes (such as bodily expressions and speech). 
The advantage of describing actions as a text is that during the execution of the pre-programmed scenario, one can add new actions while training and therefore it is possible to easily personalize or optimize training scenario.

\section{Combining the state concept with the flow of constant sensory update for easy creation of dynamic robot behaviors}

As previously said, the drawbacks of most graphical programming environments are that they can only deal with rather simple problems. For this purpose in TiViPE within the constant flow of sensory information, a state space concept has been created. The main advantage of using states within a behavior, an active state or a set of states is that state transitions can be set up like solving a tiny task or problem. Once this task is accomplished, the scenario proceeds to the next state or the set of states. The state chart could translate the end-users idea of dynamic behavior (how to go from state to state and back) to a robot dynamic behavior. As a result, the behavior of the robot continues naturally even after unexpected disturbances from the environment occur. The real-time update of the sensory information makes it possible to have a constant feedback on the ongoing robot actions. For instance, when a robot tries to grasp an object it might drop it. The robot will repeat the action of grasping, but since the object has probably fallen at a different position, the robot has to recognize this new position.

In addition, the pace of the sensory and behavioral level of information processing differs a lot. Even the simplest behavior is magnitudes longer than the sensing. In the TiViPE environment the robot sensors are permanently read out and (partly) processed. The sensing takes place in parallel to processing of (textually defined) action commands that are executed on that robot. The sensing is mostly performed on regular update speeds of up to $500 \mathrm{~ms}$ and can therefore be one order of magnitude faster than the robot action commands could be executed. This implies that the robot is not always ready to receive a new set of action commands until the previous commands have been executed. To gain an optimal responsiveness, the length of the set of actions should be minimized. The sensing, perceiving, and acting cycle is done therefore in parallel, as recommended in [24, 25]. Constructing behaviors is accomplished by coupling sets of actions by adopting the state chart concept [26], combined with the flow of constantly updating of all the sensory information available to the robot in the graphical flowchart of this behavioral sequence.

\section{Hiding Complexity}

Once a sequence of behaviors or a state chart of behavioral components is detected to be often used in combination, a simple marking of these components compiles them to a single behavioral block. By the creation of state diagrams, the end-user does not see the sensory flow, only defines the sequences of actions. We are wondering whether such a translation is easy to be automated.

A set of scenarios with specific learning goals was co-developed with the therapists. This is the necessary content for the platform to use during training sessions with the robot and a child. 


\section{$3 \quad$ User Testing Method}

The therapists play a crucial role in the several design iterations which were performed. This includes designing robot scenarios and behaviors, conducting user tests and evaluations of these and making necessary improvements. We evaluate each iteration phase using the formative evaluation method of the Cognitive Dimensions Framework (CDF). The dimensions that were used and its interpretations taken directly from the manual [22] have the following meaning: The Cognitive Dimension "Abstraction" is interpreted as the types and availability of abstraction mechanisms, "Hidden dependencies" has the meaning that important links between entities are not visible; "Premature commitment " constraints on the order of doing things, "Secondary notation" means extra information ( other than formal syntax); "Viscosity" refers to resistance to change; "Visibility" is the ability to view components easily; "Closeness of mapping: is the closeness of representation to domain; "Consistency" means that similar semantics is expressed in similar syntactic forms; "Diffuseness" refers to the verbosity of language; "Error-proneness" notation inventarises mistakes; "Hard mental operations" - high demand on cognitive resources; "Progressive evaluation" means that work-to-date can be checked at any time; "Provisionality" is the degree of commitment to actions or marks; and "Role-expressiveness" denotes that the purpose of a component is readily inferred.

In the formative evaluation we use the cognitive dimensions to give name to difficult to quantify concepts. This makes it easier to compare different concepts and make trade-offs. In this paper, we elaborate on the first evaluation which was a study in two clinics. Five participants from the first clinic and three from the second clinic took part of the experiment.

In the evaluation, the participants were given four different tasks, based on the different types of user activity described in the Cognitive Dimensions Framework, defined as incrementation, modification, transcription and exploratory design. The tasks that we created were respectively: Adding a component, editing a component, adding a new section to the network and making an own contribution to the network. For the tasks, a scenario was provided in flowchart form on paper, and a network representing this scenario was also pre-made. The participants had to complete and use this scenario and network during the four tasks.

In this first evaluation, all participants had their first experience with the programming environment. We described the first task extensively, and each following step with less detail. This gave our subjects the opportunity to quickly become familiar with the functionalities of the program, which gives them the freedom to explore in the final test, where they had to make their own contribution.

The programming environment was not demonstrated beforehand. Instead, a short explanation on paper was provided describing components and how these are connected, along with the task list and flowchart. Participants were asked to think aloud while performing the tasks, and were free to ask questions when they became stuck.

After the tasks the experience of the participants were evaluated with a short questionnaire (in Dutch for our participants). The questionnaire featured 28 statements on 
their experience with components and network in TiViPE, and 7 statements about how the robot was perceived. There was extra room for comments at the end of the questionnaire. Statements could be answered with a five-point Likert scale (fully don't agree, don't agree, neutral, do agree, fully agree). In this format, it was possible for the participants to quickly give their opinion about their experience. The statements all related to one or more of the cognitive dimensions. We also asked the participants for their experience level with other computer tasks on a five point scale (Basic, below average, average, above average, expert; each level had a description with examples).

The goal of the pilot study was to make sure the questionnaire and tasks were defined properly and was performed with 5 people from different healthcare backgrounds (four female and one male, average age 33, computer skill level was marked from basic to above average). After the pilot, the questionnaire and task list were improved to make the different dimensions in the questionnaire more balanced, and improved the wording.

Three therapists from the second clinic were then invited to go through the tasks and questionnaire. They were all females aged between 24 and 27, and had a higher educational degree than the pilot subjects. They all marked above average on the computer skill level inquiry. The setup with the robot, programming environment and instructions is shown in Figure 1.

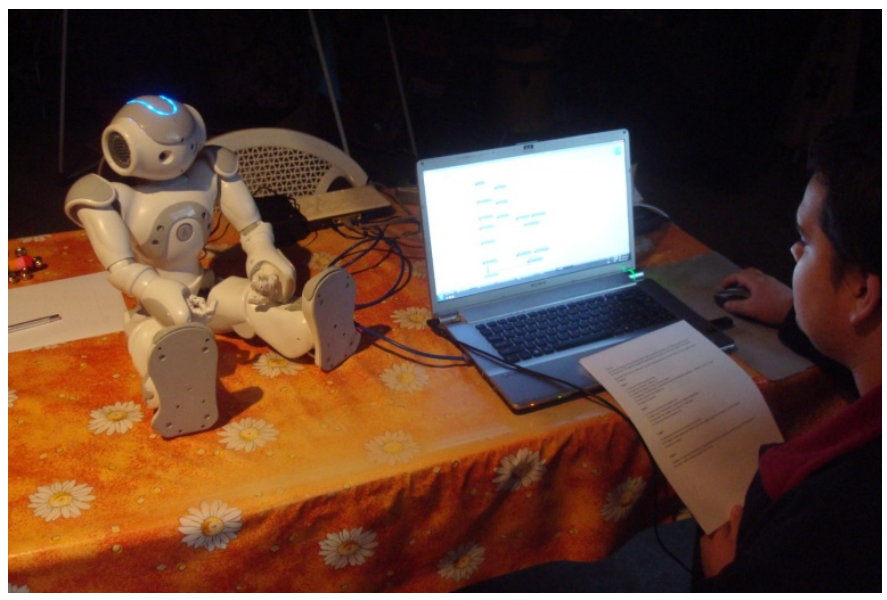

Fig. 1. Experimental setup

\section{$4 \quad$ Results}

Results from the questionnaires were compared with the cognitive dimensions each question represented. Together with the qualitative data, statements can then be made about the different dimensions and their trade-offs. 
The therapists in the second clinic performed the four tasks faster than the pilot test group (between 15 and 20 minutes compared to 20 to 25 minutes) The difference in training of the therapists from both clinics was that in the first one.

Each participant had a different approach to the final exploratory task. In the group of the second clinic, one subject would add extra components; a second altered the existing components, and a third started looking for other components that were not part of the original scenario.

During both pilot tests, it was observed that the subjects required extra help at the start (first one or two tasks), but quickly managed to use the basic functionalities of the program. There was a need for clear instructions at the start, but each participant was able to do the final task on their own.

The results from the questionnaire were gathered and mapped on the different cognitive dimensions (Table 1). Since a small sample was used, the high scores were marked with good or very good, and neutral scores with moderate. No noticeable negative attitudes towards a specific cognitive dimension were observed. Two dimensions scored noticeably higher than the other ones: role expressiveness (how self-explanatory are the different elements?) and provisionality (how accurately are the user's actions performed?). This means that the users could clearly see how each component of a program relates to the complete robot behavior and that users could with little effort rich accuracy by implementation of the scenario changes and adaptations.

Table 1. Results questionnaire in Cognitive Dimensions

\begin{tabular}{|l|l|}
\hline Cognitive Dimension & Score from Questionnaire \\
\hline Abstraction & good \\
\hline Secondary Notation & moderate \\
\hline Viscosity & good \\
\hline Consistency & good \\
\hline Error Proneness & moderate \\
\hline Progressive Evaluation & good \\
\hline Provisionality & very good \\
\hline Hidden Dependencies & good \\
\hline Premature Commitment & moderate \\
\hline Visibility & good \\
\hline Hard Mental Operations & moderate \\
\hline Closeness of Mapping & good \\
\hline Diffuseness & good \\
\hline Role-Expressiveness & very good \\
\hline
\end{tabular}


In both tests the cognitive translation from flowchart to TiViPE network was not straightforward. It took some effort to see the connection between the flowchart model, and the component network. This can be linked to the hard mental operations and abstraction dimensions.

Some mistakes were made with connecting components during the first task, which required precision in clicking sertain areas. This could affect the error proneness, and something that could be improved in the interface. This shortcoming was compensated with different other dimensions, such as provisionality. Decreasing the possibility to make errors can also decrease the speed one can work with the program, or how much control one has while building a scenario.

Within this analysis one has to note that the Cognitive Dimensions framework gives a broad- brush usability profile, intended to provide discussion tools, rather than detailed metrics [20].

\section{Additional usability test with adolescents with autism}

As part of a summer camp, two groups of 4 and 5 adolescents (age 16-18, accompanied by their caretakers) visited the robotics lab at the university. We organized an interactive session for them where they got a presentation about the project and the robot, and were then given the opportunity to create a short scenario and perform it together with the robot.

To make sure we would be able to set up a reliable short robot-adolescent interaction within minutes, we used only simple building blocks, i.e. a robot statement and a touch sensor. Still with these blocks two different stories were developed by the two groups. The first one was the robot functioning as a friendly alarm clock. The second was the robot reminding you to take your medication on time.

In both sessions, we asked the adolescents for a volunteer to do the programming in our visual programming environment. We were surprised by how quickly they were able to connect and adapt the different components on their own, which are the two core principles of our program.

\section{Conclusion}

The results from the evaluation give a positive preliminary results that support our hypothesis that by providing the appropriate design of the user-centered platform and formalizing the process of content creation, high-tech platforms as robots can be used for training children with autism.

The evaluation has been done by utilizing on the flow-chart concept for formalizing robot interaction behaviors only. The therapists were familiar with translating a child learning goal to flow-chart based scenario. Since we aim to introduce more complex and interactive scenarios, it is important how the novel to the therapists and technically more complex concepts are presented, while keeping the interaction with the program simple. The positive results from the cognitive dimensions analysis show that there is a good starting point, and from the qualitative data, we conclude that the participants can handle more complexity. 
Creating scenarios that incorporate parallel commands, task scheduling and state concepts are not tested yet. Creating several different scenarios will make possible to formalize elements of the process of scenario creation with the aim to further automate it. In addition, although the programming concepts can be presented in an understandable way within TiViPE and most of the complexity is hidden due to its design, the interface of this tool still appears complex for end users and additional layer if an interface is necessary. We have created simple interface for the control of the robot during therapy sessions, which displays what the robot says and what are the response possibilities of the experimenter or therapist.

We assume that personnel within the clinics are becoming more familiar with computer interfaces. This might further lower the hurdle to work with novel computer interfaces and the upload of training content by non-specialists. Presentation of the information for the participants was important. They mentioned that the handouts with information were very useful, but we can take this a step further, for example with video tutorials.

The sessions with adolescents showed that older adolescents had a keen interest in programming the robot, and were able to contribute in co-designing short scenarios. It may be worth exploring further whether designing social behaviors by autistic adolescents can be beneficial for their development and helpful for the development process of the training program as well since these adolescents understand the thinking patterns of autistic children.

\section{References}

1. Giullian, N., Ricks, D., Atherton, A., Colton, M., Goodrich, M., Brinton, B.: Detailed requirements for robots in autism therapy. In: 2010 IEEE International Conference on Systems Man and Cybernetics (SMC), pp. 2595-2602 (2010)

2. Kozima, H., Nakagawa, C.: Interactive Robots as Facilitators of Children' s Social Development. In: Mobile Robots: Toward New Applications, pp. 269-286 (December 2006)

3. Robins, B., Dickerson, P., Stribling, P., Dautenhahn, K.: Robot-mediated joint attention in children with autism: A case study in robot-human interaction. Interaction Studies 5(2), 161-198 (2004)

4. Bird, G., Leighton, J., Press, C., Heyes, C.: Intact automatic imitation of human and robot actions in autism spectrum disorders. Proceedings of the Biological sciences / The Royal Society 274(1628), 3027-3031 (2007)

5. Brok, J.C.J., Barakova, E.I.: Engaging Autistic Children in Imitation and Turn-Taking Games with Multiagent System of Interactive Lighting Blocks. In: Yang, H.S., Malaka, R., Hoshino, J., Han, J.H. (eds.) ICEC 2010. LNCS, vol. 6243, pp. 115-126. Springer, Heidelberg (2010)

6. Dautenhahn, K., Werry, I.: Towards interactive robots in autism therapy. Pragmatics \& Cognition 1(12), 1-35 (2004)

7. Duquette, A., Michaud, F., Mercier, H.: Exploring the use of a mobile robot as an imitation agent with children with low-functioning autism. Autonomous Robots 24(2), 147-157 (2008) 
8. Pioggia, G., Igliozzi, R., Sica, M., Ferro, M., Muratori, F.: Exploring emotional and imitational android-based interactions in autistic spectrum disorders. Journal of CyberTherapy \& Rehabilitation 1(1), 49-61 (2008)

9. Robins, B., Dautenhahn, K., Te Boekhorst, R., Billard, A.: Robotic assistants in therapy and education of children with autism: can a small humanoid robot help encourage social interaction skills? Universal Access in the Information Society 4(2), 105-120 (2005)

10. Barakova, E.I., Lourens, T.: Expressing and interpreting emotional movements in social games with robots. Personal and Ubiquitous Computing 14, 457-467 (2010)

11. Barakova, E.I., Gillesen, J., Feijs, L.: Social training of autistic children with interactive intelligent agents. Journal of Integrative Neuroscience 8(1), 23-34 (2009)

12. Feil-Seifer, D., Mataric', M.: Robot-assisted therapy for children with autism spectrum disorders. In: Proceedings of the 7th International Conference on Interaction Design and Children, IDC 2008, vol. (2005), p. 49 (2008)

13. Gillesen, J.C.C., Barakova, E.I., Huskens, B.E.B.M., Feijs, L.M.G.: From training to robot behavior: Towards custom scenarios for robotics in training programs for ASD. In: IEEE International Conference on Rehabilitation Robotics, pp. 387-393 (2011)

14. Bernd, T., Gelderblom, G.J., Vanstipelen, S., de Witte, L.: Short term effect evaluation of IROMEC involved therapy for children with intellectual disabilities. In: Ge, S.S., Li, H., Cabibihan, J.-J., Tan, Y.K. (eds.) ICSR 2010. LNCS, vol. 6414, pp. 259-264. Springer, Heidelberg (2010)

15. Colton, M.B., Ricks, D.J., Goodrich, M.A., Dariush, B., Fujimura, K., Fujiki, M.: Toward Therapist-in-the-Loop Assistive Robotics for Children with Autism and Specific Language Impairment. In: AISB New Frontiers in Human-Robot Interaction Symposium, vol. 24, p. 25 (2009)

16. Hume, K., Bellini, S., Pratt, C.: The Usage and Perceived Outcomes of Early Intervention and Early Childhood Programs for Young Children With Autism Spectrum Disorder. Topics in Early Childhood Special Education 25(4), 195-207 (2009)

17. Simpson, R.L.: Evidence-Based Practices and Students With Autism Spectrum Disorders. Focus on Autism and Other Developmental Disabilities 20(3), 140-149 (2005)

18. Harris, S.L., Delmolino, L.: Applied Behavior Analysis: Its application in the Treatment of Autism and Related Disorders in Young Children. Infants \& Young Children 14(3), 11-17 (2002)

19. Koegel, R.L., Koegel, L.K.: Pivotal Response Treatments for Autism: Communication, Social and Academic Development, p. 296. Brookes Publishing Co. (2006)

20. Koegel, R.L., Koegel, L.K., McNerney, E.K.: Pivotal areas in intervention for autism. Journal of Clinical Child \& Adolescent Psychology 30(1), 19-32 (2001)

21. Lourens, T.: TiViPE-Tino's Visual Programming Environment. In: The 28th Annual International Computer Software \& Applications Conference, IEEE COMPSAC, pp. 10-15 (2004)

22. Green, T., Blackwell, A.: Cognitive dimensions of information artefacts: a tutorial. In: BCS HCI Conference (October 1998)

23. Barakova, E.I., Gillesen, J.C.C., Huskens, B.E.B.M., Lourens, T.: End-user programming architecture facilitates the uptake of robots in social therapies. Robotics and Autonomous Systems 61, 704-713 (2013)

24. Gibson, J.: The Senses Considered as Perceptual Systems. Houghton-Mifflin, Boston (1966)

25. Barakova, E.I., Lourens, T.: Mirror neuron framework yields representations for robot interaction. Neurocomputing 72(4-6), 895-900 (2009) 
26. http://www.tivipe.com/index.php?option=com_content\&view=arti cle\&id=90: robot-throwing-dice\&catid=53: tivipe-use-andapplications\&Itemid $=90$

27. Giannopulu, I.: Embedded multimodal nonverbal and verbal interactions between a mobile toy robot and autistic children. Presented at the Proceedings of the 8th ACM/IEEE International Conference on Human-Robot Interaction, Tokyo, Japan (2013)

28. Robins, B., Dautenhahn, K., Ferrari, E., Kronreif, G., Prazak-Aram, B., Marti, P., Iacono, I., Gelderblom, G.J., Bernd, T., Caprino, F.: Scenarios of robot-assisted play for children with cognitive and physical disabilities. Interaction Studies 13, 189-234 (2012)

29. http://www.idemployee.id.tue.nl/e.i.barakova/ 